\title{
Fractionalization, topological order, and cuprate superconductivity
}

\author{
T. Senthil and Matthew P.A. Fisher \\ Institute for Theoretical Physics, University of California, Santa Barbara, CA 93106-4030
}

(October 31, 2018)

\begin{abstract}
This paper is concerned with the idea that the electron is fractionalized in the cuprate high- $T_{c}$ materials. We show how the notion of topological order may be used to develop a precise theoretical characterization of a fractionalized phase in spatial dimension higher than one. Apart from the fractional particles into which the electron breaks apart, there are non-trivial gapped topological excitations - dubbed "visons". A cylindrical sample that is fractionalized exhibits two disconnected topological sectors depending on whether a vison is trapped in the "hole" or not. Indeed, "vison expulsion" is to fractionalization what the Meissner effect ("flux expulsion") is to superconductivity. This understanding enables us to address a number of conceptual issues that need to be confronted by any theory of the cuprates based on fractionalization ideas. We argue that whether or not the electron fractionalizes in the cuprates is a sharp and well-posed question with a definite answer. We elaborate on our recent proposal for an experiment to unambiguously settle this issue.
\end{abstract}

\section{INTRODUCTION}

The cuprate high- $T_{c}$ materials are amongst the most complicated systems studied extensively in solid state physics. In addition to the high temperature superconductivity itself, they display a wide variety of novel phenomena. Perhaps the most puzzling is the behaviour in the "normal" non-superconducting state above the transition temperature which, being anything but normal, is difficult to understand within Fermi liquid theory. The superconductivity is obtained by doping "parent" compounds that are Mott insulators - rendered insulating by strong electron-electron interactions. These parent compounds also display Neel antiferromagnetism. A number of other interesting phases and broken symmetries are also often observed, including charge and spin ordering into stripes. In addition, some regions of the phase diagram are very sensitive to the presence of disorder particularly at low doping and low temperature. Indeed, even a casual glance at the phase diagram is sufficient to realize the richness of phenomena displayed by these materials.

It is hoped by many that underlying this remarkably complex behaviour, might lie a simple explanation which will give insight into the mechanism of the superconductivity. The challenge is to identify any key qualitative features of the system which can be sharply characterized and detected experimentally. In this paper, we pursue an elegant and simple explanation of the superconductivity and other properties that is based on the idea that the electron is splintered apart (i.e fractionalized) in these materials. The genesis of this idea can be traced back to the original RVB theories [1 [3], but recent theoretical work [4] has lead to a unified theoretical framework for electron fractionalization above one spatial dimension (most readily expressed in terms of a $Z_{2}$ gauge theory $[7,(\beta])$. Remarkably, this points to a novel route to superconductivity which dispenses entirely with the notion of electron pairing. Quite generally, to obtain superconductivity in a many-body system it is necessary to condense a charged particle. In an electronic system the naive route would be to condense the electron, but this is of course not possible as the electron is a fermion. The BCS solution was to argue that a weak attractive interaction between the electrons (or more precisely between Landau quasiparticles) binds them into pairs, which condense as a charge $2 e$ boson. But fractionalization describes an altogether different route to superconductivity, within which the electron splinters into two pieces, one carrying the Fermi statistics (and spin) - a direct condensation of the remaining charge $e$ boson leads directly to superconductivity. Remarkably, although the fractionalization route to superconductivity is so very different from that in BCS theory, the resulting superconducting phase itself has identical qualitative properties [7.9. Furthermore, the fractionalization idea provides appealing explanations of several of the unusual "normal" state phenomena, most notably the photoemission spectra.

In this paper, we show how a precise meaning may be given to the statement that the electron is fractionalized. Based on this, we argue that whether or not the electron fractionalizes in the cuprates is a sharp theoretical question that is independent of all kinds of unavoidable material complications. Further, we show how this sharp theoretical question may be answered unambiguously by experiments. The idea that the electron is fractionalized thus provides a non-pairing route to superconductivity which is directly testable.

We begin by developing a precise theoretical characterization of a phase in which the electron is fractionalized. As anticipated in Ref. [5], this is through the notion of "topological order" - a concept that has been elucidated clearly by Wen and coworkers [10] in the context of the quantum Hall effect. This enables us to address a number of conceptual issues that need to be confronted by any theory of the cuprates based on fractionalization ideas. The crucial property of the fractionalized phase is the ex- 
istence of excitations which are fractions of the electron. While various such phases with different fractionalization patterns are theoretically possible 11, the phase that is of the most interest in the context of the cuprates is one in which the electron breaks into a charged boson and a neutral spin carrying fermion. An equally crucial property of the fractionalized phase is the emergence of a gapped topological excitation - dubbed the vison [7]. A pair of visons can annihilate each other, so that they carry only a $Z_{2}$ (topological) quantum number. The existence of this topological excitation is conceptually very important to the "fractionalization" route to superconductivity. The superconductor obtained by condensing the charge of an electron (once having shed it's Fermi statistics) is in the same phase as the one obtained by condensing Cooper pairs of electrons. In particular, despite the condensation of a charge $e$ boson, flux quantization is in units of $h c / 2 e$ - this surprising result [12,7] requires the presence of the topological vison excitations in the fractionalized phase. Indeed, the visons bind to an $h c / 2 e$ unit of electromagnetic flux once the system becomes superconducting.

Any complete theory of the cuprates must necessarily pay attention to their layered quasi-two dimensional structure. Motivated by this, we consider the possible fractionalized phases in such a geometry. Interestingly, two qualitatively distinct kinds of fractionalized phases are possible. In one, the system behaves as a full three dimensional solid, and the fractions into which the electron decays can freely propagate in all three directions. In the other, the different layers decouple from each other - the fractions of the electron can now propagate freely within each layer but cannot do so in the direction perpendicular to the layers. A number of experiments suggest that this decoupled quasi- $2 d$ fractionalized phase is the one more likely relevant to the cuprates.

Another important issue is the fate of the fractionalization at finite temperature [7]. One normally associates fractionalization with a property of the spectrum of the system's Hamiltonian - it is therefore not a priori clear whether it has any meaning at finite temperature. However, having characterized the fractionalized phase by it's topological order (rather than by it's spectrum) we are able to address this issue. For the quasi-2d fractionalized phase, the topological order in fact does not survive at finite temperature, so that a sharp distinction between fractionalized and unfractionalized phases is only possible at zero temperature. Nevertheless, at low temperature above the fractionalized phase, the system is "almost" topologically ordered. In the cuprates, we have suggested [9] that the crossover towards the $T=0$ fractionalization occurs at a temperature comparable to the pseudogap temperature. As we will see, this may be exploited to probe the hidden zero temperature order in the system. (For the fully three dimensional fractionalized phase, on the other hand, the topological order survives up to a finite non-zero temperature [7]).

If fractionalization occurs at all in the cuprates, it is most likely in the underdoped regime. This might appear to raise serious problems for the fractionalization idea, since it is precisely in the heavily underdoped region at low temperature that a variety of conventional broken symmetry states (Neel magnetism, or charge and spin stripes) are observed. Furthermore, this region tends to be very sensitive to disorder effects. We argue that this is a non-issue. Theoretically, the topological order that characterizes fractionalization can happily co-exist with Neel magnetism [13], or stripes, or other broken symmetry states. Moreover, it is unaffected by disorder. Thus, the presence of a conventional broken symmetry tells us nothing about whether or not the system is fractionalized at zero temperature. If the electron is indeed fractionalized in the underdoped cuprates, the conventional ordered states seen in that region are complications that distract from the hidden zero temperature topological order that is ultimately responsible for the superconductivity.

Historically, theoretical attempts to access fractionalized phases above one dimension have focused on "quantum disordering" various states with conventional wellunderstood broken symmetries, most frequently antiferromagnets and superconductors. This has led to a misconception that fractionalization requires the close proximity to a "parent" conventional broken symmetry state. This, however, is both problematic and incorrect. Clearly there can be "quantum disordered" magnets or superconductors which are not fractionalized. Moreover, ordered phases which are fractionalized are certainly possible, at least in principle. As we emphasize in this paper, the correct way to characterize any fractionalized phase is by specifying it's topological order. However, the fractionalized phase does often contain in it the seed of broken symmetry, particularly in electronic systems. For example, once the electron charge (or spin) has been liberated from it's Fermi statistics, a direct condensation leads naturally to superconductivity (or magnetism). But note, here the broken symmetry emerges from the fractionalization - the latter being the higher energy phenomenon. For instance, if fractionalization occurs at all in the cuprates, the energy scale is presumably comparable to the pseudo-gap temperature [9] - and the superconductivity is an emergent low energy phenomenon. Thus it is more correct to view the fractionalized phase as the "parent" phase to the broken symmetry state - rather than the other way around.

While the underdoped cuprates are possibly fractionalized, the empirical evidence seems to suggest that when heavily overdoped they are not. As we have detailed earlier [9], the quantum confinement transition where the fractions of the electron get glued back together might well account for the properties in the region between the underdoped and overdoped regimes. A complete theory of this novel quantum phase transition is unfortunately 
unavailable at present - we instead will briefly discuss some much simpler quantum confinement transitions.

Most importantly, the theoretical understanding of fractionalization developed in this paper enables us to describe an experimental setup which should enable a direct detection of the topological order. As we shall see, the hallmark of fractionalization is the expulsion of visons - analogous to the Meissner effect being the hallmark of superconductivity. We describe a way to prepare and detect a vison in the hole of a cylindrical sample. If the "normal state" of the underdoped cuprates is fractionalized, and hence topologically ordered, the trapped vison will be unable to escape, and can be detected at a later time. This signature of fractionalization in the "normal state", is directly analogous to fluxoid trapping in a superconductor. Some of the results of this paper, mainly the proposal for the experiment described above, were briefly presented in a recent short paper [14].

In the rest of the paper, we elaborate on the ideas and results described above. The theoretical formulation we use to describe fractionalization is a $Z_{2}$ gauge theory. While this is mathematically and physically closely related to several other formulations, it has several advantages. It works directly with the physical excitations in the fractionalized phase. Moreover, the topological order characterizing the fractionalized phases is most simply discussed in the $Z_{2}$ gauge theory framework. It also has the advantage that it generalizes readily to a variety of relevant situations, such as layered systems or a system with broken spin rotation invariance.

\section{FRACTIONALIZATION AND TOPOLOGICAL ORDER}

\section{A. Review of $Z_{2}$ formulation}

In our recent work 77 we demonstrated that a general class of strongly interacting electron models could be recast in the form of a $Z_{2}$ gauge theory, which then enabled us to provide a reliable discussion of issues of electron fractionalization. In particular, we demonstrated the possibility of obtaining fractionalized phases in two or higher spatial dimensions. We begin with a quick review of this formulation.

The action for the $Z_{2}$ gauge theory is

$$
\begin{aligned}
S & =S_{c}+S_{s}+S_{K}+S_{B} \\
S_{c} & =-t_{c} \sum_{\langle i j\rangle} \sigma_{i j}\left(b_{i}^{*} b_{j}+c . c .\right) \\
S_{s} & =-\sum_{\langle i j\rangle} \sigma_{i j}\left(t_{i j}^{s} \bar{f}_{i \alpha} f_{j \alpha}+t_{i j}^{\Delta} f_{i \uparrow} f_{j \downarrow}+c . c\right)-\sum_{i} \bar{f}_{i \alpha} f_{i \alpha} \\
S_{K} & =-K \sum_{\square} \prod_{\square} \sigma_{i j} .
\end{aligned}
$$

Here, $b_{i}^{\dagger}$ creates a spinless, charge $e$ bosonic excitation the chargon - and $f_{i}^{\dagger}$ creates the spinon, a fermion carrying spin $1 / 2$ but no charge. When created together, these two excitations comprise the electron. The field $\sigma_{i j}$ is a gauge field that lives on the links of the space-time lattice (taken as cubic when in $2+1$-dimensions), and takes on two possible values: $\sigma_{i j}= \pm 1$. The kinetic term for the gauge field, $S_{K}$, is expressed in terms of plaquette products. Here, $S_{B}$ is a Berry's phase [7] term which depends on the doping $x$.

At a formal level, the action above reformulates a system of interacting charge $e$, spin $1 / 2$ electrons as a system of spinless, charge $e$ bosons (the chargons) and neutral, spin 1/2 fermions (the spinons) both of which are minimally coupled to a fluctuating $Z_{2}$ gauge field. The physical content of any gauge field is in it's vortex excitations that carry the gauge flux. We are therefore led to consider vortices in the $Z_{2}$ gauge field - dubbed the "vison". Specifically, consider the product of the gauge field $\sigma$ around an elementary plaquette, which can take on two values, plus or minus one. When this product is negative, a vison excitation is present on that plaquette.

We may therefore regard the action in Eqn. 11 above as a reformulation of an interacting electron system as a theory of interacting chargons, spinons, and visons. At this stage, this is essentially nothing more than a change of variables on the original electronic system. However this reformulation is an extremely useful starting point to discuss phases of the system where the electron is fractionalized. Both the chargons and spinons carry a unit of $Z_{2}$ gauge charge while the vison carries a unit of $Z_{2}$ gauge flux. Thus, upon encircling a vison, the chargon and spinon each acquire a phase of $\pi$. This long range interaction has crucial implications for the physics.

There are two qualitatively different phases that are described by the $Z_{2}$ gauge theory action. In one, the visons are gapped excitations. In such a phase, the electron splits into two independent excitations - the chargons and the spinons. To see this simply, consider the limit when the vison gap is very large so that they may be safely ignored (ie. $K \rightarrow \infty$ ). Thus, when the visons are absent, all the plaquette products of the $Z_{2}$ gauge field equal plus one. One can therefore put $\sigma_{i j}=1$ on every link. In this case the chargon and spinon can propagate independently, and the electron is fractionalized.

The other qualitatively different kind of phase is obtained if the visons are condensed. The long range interaction between the visons and the chargons (or the spinons) frustrates the motion of the latter. The result is that they are confined together to form electrons (or other composite excitations made out of electrons). In such a phase, the electron is not fractionalized. Further, once the vison is condensed, it loses it's legitimacy as an excitation in the system. 
Thus the really crucial property of the fractionalized phase is the presence of the gapped topological vison excitations. The full excitation spectrum in the fractionalized phase decomposes into different topological sectors. The fractionalized phase is therefore characterized by the emergence of a topological quantum number which labels the spectrum of states. Topological excitations are also well-known to occur in states with a broken symmetry - for instance, vortices in superconductors. However the topological excitations in the fractionalized phase occur despite the absence of any obvious broken symmetry.

Nevertheless, the fractionalized phase contains in it the seed of broken symmetry. Once the electron is splintered into the chargon and the spinon, it's electric charge is no longer tied to it's Fermi statistics. Instead, the charge is now carried by the bosonic chargons. The chargons can now directly condense leading to a superconducting state. Surprisingly, this superconductor is in the same phase as that obtained by the condensation of Cooper pairs of electrons. In particular, the superconductor has flux quantization in units of $h c / 2 e$ despite it's description as a condensate of charge $e$ chargons. This remarkable feature is due to the presence of the topological excitations - the visons - in the fractionalized phase. Indeed upon condensing the chargon to form the superconductor, the vison also acquires $h c / 2 e$ of electromagnetic flux.

In the rest of this section, we will develop a precise theoretical characterization of the fractionalized phase using the notion of topological order.

\section{B. Topological order in the pure gauge theory}

We begin by considering the pure gauge theory in the absence of any matter coupling (i.e coupling to the chargons or the spinons). This is described by the action

$$
S_{K}=-K \sum_{\square} \prod_{\square} \sigma_{i j} .
$$

For concreteness, we specialize to a two-dimensional spatial square lattice, plus one time dimension. It will often also be convenient to consider the equivalent quantum Hamiltonian [15] in two spatial dimensions:

$$
H=-K \sum_{\square} \prod_{\square} \sigma_{r r^{\prime}}^{z}-h \sum_{<r r^{\prime}>} \sigma_{r r^{\prime}}^{x}
$$

Here $r, r^{\prime}$ label the sites of the $2 d$ square lattice and $\sigma_{r r^{\prime}}^{z}, \sigma_{r r^{\prime}}^{x}$ are Pauli matrices that live on the bonds of the lattice. The first term involves products over spatial plaquettes only.

It is well-known [15] that this pure $Z_{2}$ gauge theory has two phases. For $K$ small, there is a phase where static test charges that couple to the gauge field are confined. For $K$ large, on the other hand, there is a different phase where such test charges are allowed to be deconfined.
This distinction may be quantified by the behaviour of the "Wilson loop" correlator [15] - this decays exponentially with the area of the loop in the small $K$ phase, but only with the perimeter in the large $K$ phase.

A different, but equivalent, view of these two phases is in terms of the vison excitation i.e, the vortex of the $Z_{2}$ gauge field. In the perimeter law phase, the vison is a gapped excitation. In the area law phase, on the other hand, the vison is condensed. This can be understood very explicitly by means of a duality transformation [15.16] to the global Ising model described by the Hamiltonian:

$$
H=-h \sum_{r r^{\prime}} v_{r}^{z} v_{r^{\prime}}^{z}-K \sum_{r} v_{r}^{x}
$$

This global Ising model is defined on the lattice dual to the original square lattice. The $v_{r}^{z}, v_{r}^{x}$ are also Pauli matrices. The dual Ising spin $v_{r}^{z}$ has the physical interpretation of being the vison creation operator [15, (7). For small $K$, the global Ising model is in it's ordered state, and the visons are therefore condensed. For large $K$, on the other hand, the global Ising model is in it's disordered phase, and the visons are gapped.

The two phases of the gauge theory Hamiltonian in Eqn. 6 may be distinguished in yet another way - this is through the notion of "topological order". Consider the gauge theory Hamiltonian on a manifold with a nontrivial topology. In the deconfined (large $K$ ) phase, as we discuss at length below, the ground state has a degeneracy (in the thermodynamic limit) which depends on the topology of the manifold. In the confined phase on the other hand, there is a unique ground state independent of the topology of the manifold. This is a precise, and as we shall see, powerful distinction between the two phases. Such a distinction was originally pointed out for pure (non-abelian) gauge theories in pioneering work by 't Hooft [17].

This topological characterization of the phases of the gauge theory can be traced to the existence of symmetry operations specific to the topology of the manifold. These topological symmetries are preserved by the ground state in the confined phase. In the deconfined phase, these topological symmetries are spontaneously broken - this immediately leads to the ground state degeneracy on nontrivial manifolds. Such a breaking of topological symmetries also characterizes the fractional quantum Hall fluids, as expounded in some beautiful papers 10 of Wen and coworkers. Following the terminology used in that context, we will refer to the breaking of the topological symmetry as "topological order".

To fix these ideas, consider a cylindrical geometry. In the deconfined phase of the gauge theory, there are two degenerate ground states. They correspond to whether or not a vison has "threaded the hole of the cylinder" (See Fig. 1). Deep within the deconfined phase, with $K \rightarrow \infty$, 
the two corresponding gauge field configurations are very simple. Setting all of the link fields, $\sigma^{z}=1$, is clearly a ground state in this limit, and corresponds to the absence of threaded vison, since the flux of the $Z_{2}$ gauge field through any curve $C$ that encircles the cylinder,

$$
\Phi[C]=\Pi_{C} \sigma_{L}^{z}
$$

equals unity. (Here $L$ labels the bonds that belong to $C$.) The ground state with a threaded vison can be obtained, for example, by changing the sign of $\sigma^{z}$ on a column of horizontal bonds that runs the length of the cylinder (see Fig. 2) - in this state $\Phi[C]=-1$. Similarly reasoning implies that on a torus, there are four degenerate ground states corresponding to the vison threading or not threading each of the two holes. In what follows, we will analyse the cylinder in several ways to get a deep understanding of this phenomenon.
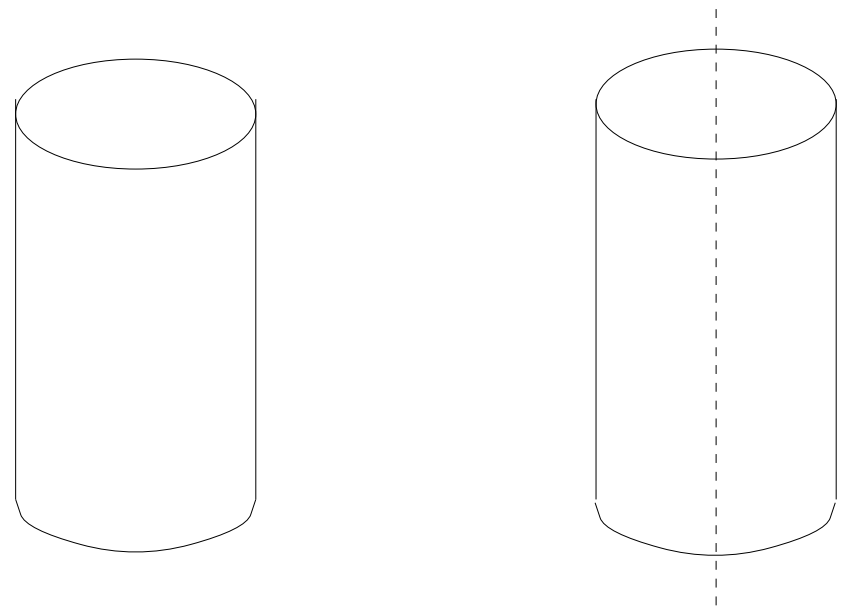

FIG. 1. The two degenerate states in a cylinder. The right one has a vison threading the "hole".

Assume that a vison is initially trapped in the hole of the cylinder when the gauge theory is in it's deconfining phase. We take the $x$ axis of space to be along the length of the cylinder, and the $y$ axis to be along the circumference. Let the length of the cylinder be $L_{x}$ and it's circumference $L_{y}$. For the vison to tunnel out, the $Z_{2}$ flux tube must penetrate the cylinder in at least two places (in general some even number), and these two points of penetration must move apart (see Fig. 3) till they drop out of the edge of the system. As there is a finite energy cost for the vison to penetrate the sample in the deconfined phase, the amplitude for this process will be exponentially small in $L_{x}$. Thus the vison tunneling rate varies as $\Gamma \sim e^{-c L_{x}}$, which goes to zero as $L_{x} \rightarrow \infty$. Thus, once trapped, a vison in the hole of the cylinder lives forever (in the thermodynamic limit).

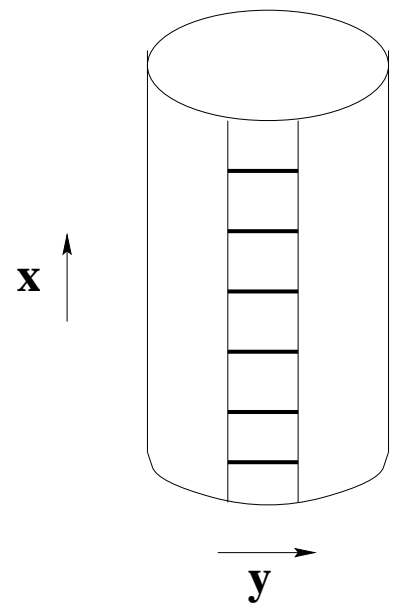

FIG. 2. Changing the sign of $\sigma^{z}$ on all the dark bonds adds (or removes) a vison from the hole of the cylinder.

Consider the situation with finite $L_{x}$ and $L_{y}$. An operator that adds (or removes) a vison from the hole can be readily constructed as follows:

$$
\mathcal{P}=\Pi_{x} \sigma_{\vec{r}, \vec{r}+\hat{y}}^{x}
$$

with $\vec{r}=\left(x, y_{0}\right)$. The operator $\mathcal{P}$ changes the sign of all the operators $\sigma_{\vec{r}, \vec{r}+\hat{y}}^{z}$ that live on the bonds (see Fig. 2) along the $y$-direction between some chosen $y$-slices $y_{0}$ and $y_{0}+1$. Consider now the flux of the $Z_{2}$ gauge field through any curve $C$ that encircles the cylinder, as defined in Eqn. 8: Clearly, $\mathcal{P}$ changes the sign of this flux. Thus $\mathcal{P}$ is an operator that adds or removes a vison from the hole of the cylinder.

It is straightforward to see that $\mathcal{P}$ commutes with the Hamiltonian Eqn. 6 of the gauge theory. Thus, it is a symmetry of the theory. Further, as it corresponds to the operation of adding a vison through the hole, it is a topological symmetry. Now consider the limit $L_{x} \rightarrow \infty$. As argued earlier, in the deconfined phase a vison that is trapped in the hole stays there forever. Consider the ground state with a vison trapped in the hole. Upon acting on this state with the operator $\mathcal{P}$, it becomes the ground state in the sector with no vison trapped. Thus, the ground state is not invariant under the action of the operator $\mathcal{P}$. The topological symmetry has been broken spontaneously. Note that the ground states in the two sectors (with or without a vison) are guaranteed to have exactly the same energy as $\mathcal{P}$ commutes with the Hamiltonian. Thus, the gauge theory in it's deconfining phase has two degenerate ground states on the cylinder. 


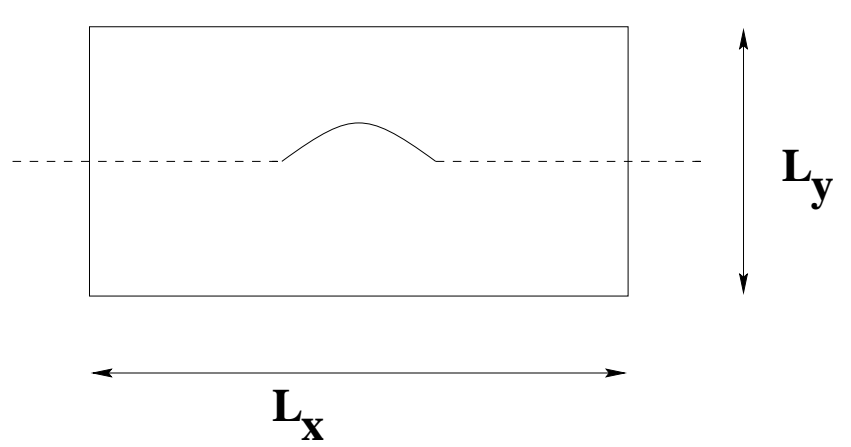

FIG. 3. Vison tunneling out of the cylinder. The dashed line represents the $Z_{2}$ flux line inside the hole. The points of penetration are where the line becomes solid. It is assumed that there are periodic boundary conditions along the $y$-direction.

Further insight into the ground state degeneracy and the broken topological symmetry is obtained by the following considerations. Imagine changing the value of $K$ to some $K_{b}$ along all plaquettes at some $y$-slice, say $y=y_{0}$. Assume that the gauge theory in the bulk is in it's deconfining phase (i.e, the bulk value of $K$ is very large). In the limit that $K$ in the bulk is $\infty$, there can be no gauge flux penetrating the bulk of the system. We may then set $\sigma^{z}=1$ for all bonds except those along the "cut". The remaining degrees of freedom live on the cut (the dark bonds in Fig. 2). The Hamiltonian describing them is clearly just a one dimensional transverse field Ising model:

$$
H=-K_{b} \sum_{x} \sigma_{x}^{z} \sigma_{x+1}^{z}-h \sum_{x} \sigma_{x}^{x},
$$

where $\sigma_{x}^{z}$ is the $Z_{2}$ gauge field on the bond at site $x$ along the cut. For small $K_{b}$, this Ising model is in it's disordered phase. The ground state is therefore unique. With increasing $K_{b}$ this edge global Ising model undergoes a phase transition to an ordered state with $\left\langle\sigma_{x}^{z}\right\rangle \neq 0$. The ground state is therefore two-fold degenerate. The two degenerate ground states correspond precisely to whether or not a vison is trapped in the hole of the cylinder. This can be seen in several ways - for instance by noting that the operator $\mathcal{P}$ introduced above is precisely the global spin flip operator of the edge Ising model. Further, the domain walls in the ordered state of the edge Ising model correspond to plaquettes where a vison has penetrated the cylinder. In the ordered phase, such domain walls, and hence the visons, cost finite energy. In the disordered state, the domain walls have proliferated - this may be interpreted as a proliferation and condensation of visons along the edge.

The phase transition discussed above is thus an edge confinement transition. The topology of the manifold in which the deconfined phase "resides" changes from a rectangle to a cylinder as the coupling $K_{b}$ is increased. We will discuss such topology-changing phase transitions further in Section VII.

Yet another route to understanding the topological ground state degeneracy of the deconfined phase is to employ the duality transformation of the full gauge theory to the global Ising model as discussed in the beginning of this subsection. For this purpose, it is convenient to consider an annulus (see Fig. 4) which is topologically equivalent to a cylinder. This can be obtained from the gauge theory defined in infinite two-dimensional space by simply setting some of the plaquette strengths to zero. First, imagine setting $K=0$ for a single plaquette in the center. This creates a "hole" in the system. Similarly, at the outer boundary of the sample, again set $K=0$ for all plaquettes. This captures the finiteness of the sample. For concreteness, we consider a circular disc of radius $R$.

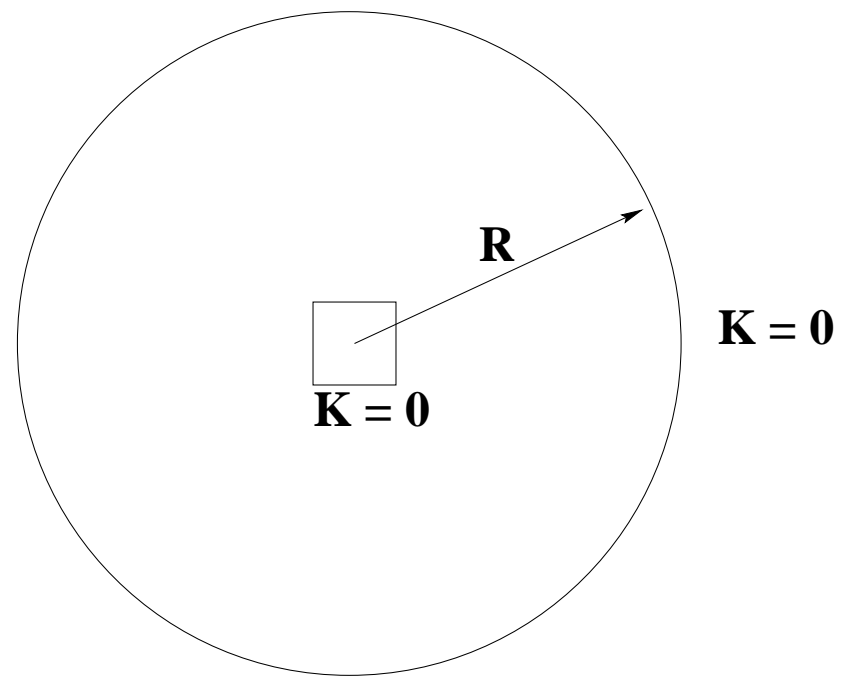

FIG. 4. The $Z_{2}$ gauge theory on an annulus with a hole at the center. This corresponds to setting $K=0$ for the "hole" plaquette, and for all the plaquettes outside the disc radius.

Now we employ the duality transformation to get a representation of the system as a global Ising model. The hole in the center of the sample goes over into a single site of the dual lattice. The restriction that $K=0$ at the hole then implies that the transverse field at this site on the dual spin is exactly zero. Similarly, at the outer boundary of the sample, $K=0$ implies that the transverse field on the dual Ising spins outside the disc radius is zero. This implies that these dual spins outside the disc radius are all lined up together 18 .

Before continuing, it is necessary to take note of one other subtle feature of the duality transformation. Two states of the dual global Ising model that only differ by an overall spin flip are not to be counted as two distinct states of the gauge theory (as may be seen from, for instance, the treatment of the duality transformation in Ref. (7]). This can be taken care of simply by fixing the direction of the frozen spins outside the disc radius to be, say, up (see Fig. 5). 


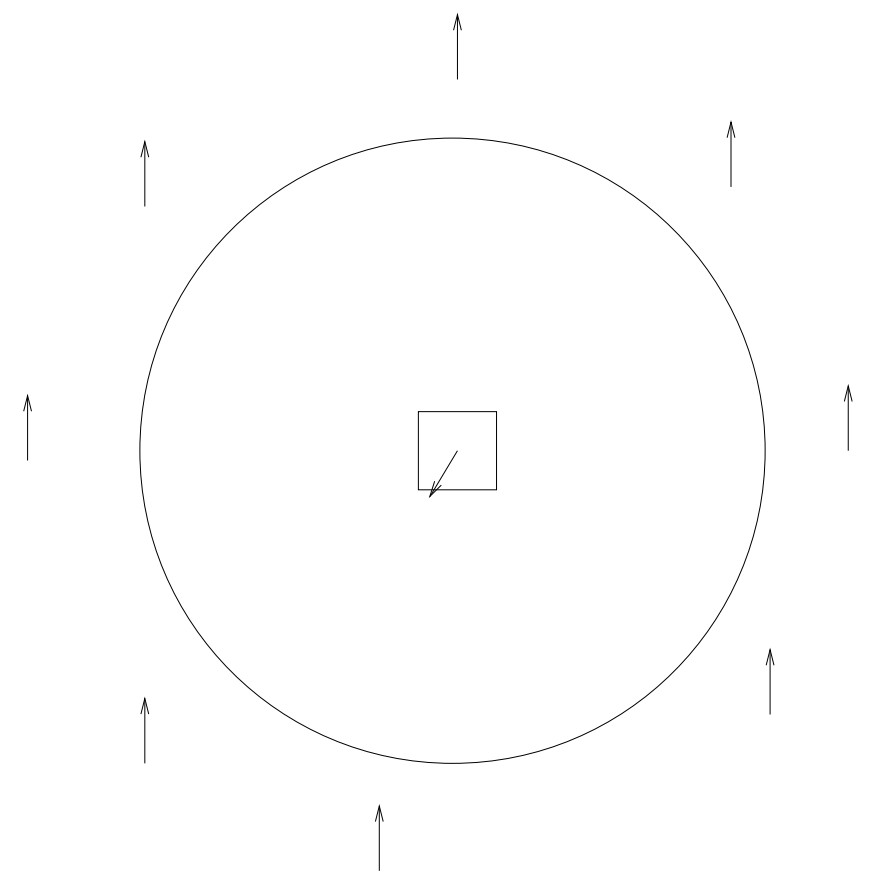

FIG. 5. The dual global Ising model on the annulus. The Ising spins outside the disc radius are frozen in the up direction. The Ising spin in the hole has no transverse field on it.

We may now discuss the ground state degeneracies of the gauge theory using the dual global Ising model. First consider the confining phase of the gauge theory. This is the ferromagnetic phase of the dual global Ising model. The direction of the boundary spins fix the direction of the ferromagnetic ordering. Thus, all the spins in the interior including the one corresponding to the hole, point in the up direction. There is no ground state degeneracy.

Now consider the deconfining phase. This corresponds to the paramagnetic phase of the dual global Ising model. The dual spin correlations decay exponentially. Thus the ordering of the boundary spins has little influence on the "hole" spin at the center. The latter is therefore essentially free to point in any direction. This then corresponds to the expected two degenerate ground states. To make this more precise, consider modelling the bulk system by a continuum scalar field theory with a Gaussian action,

$$
S=\int d \tau d^{2} x\left[(\nabla \phi)^{2}+\left(\partial_{\tau} \phi\right)^{2}+m^{2} \phi^{2}\right] .
$$

This Gaussian theory is expected to correctly describe the physics of the paramagnetic phase of the global Ising model. The coupling to the "hole" spin is through an Ising exchange term,

$$
S_{\text {hole }}=\int d \tau v^{z} \phi(\overrightarrow{0}, \tau) .
$$

Here $v^{z}$ represents the "hole" spin and we have taken the location of the hole to be at the origin. Note that the "hole" spin has no dynamics - this is due to the absence of any transverse field on that spin in the lattice model.

The action above must be supplemented with a boundary condition arising from the fixed direction of the spins outside the disc radius. This is simply the condition that

$$
\phi(\vec{x}, \tau)=\phi_{0},
$$

for $|\vec{x}|=R$ with $\phi_{0}$ a positive constant.

As the field $\phi$ is massive, it can be safely integrated out to get an effective action for $v^{z}$. For result for large $R$ is simply,

$$
S_{e f f}=2 \pi R \int d \tau \chi(R) v^{z},
$$

where $\chi(R)$ is the static susceptibility of the Ising paramagnet. This is readily computed to be

$$
\begin{aligned}
\chi(R) & =\int \frac{d^{2} q}{4 \pi^{2}} \frac{e^{i \vec{q} \cdot \vec{R}}}{q^{2}+m^{2}}, \\
& =\frac{1}{2 \pi} K_{0}(m R), \\
& \sim \sqrt{\frac{1}{8 \pi m R}} e^{-m R},
\end{aligned}
$$

where the last expression is valid for $R>>1 / m$. The effective action above for the "hole" spin may be readily converted into an effective Hamiltonian,

$$
H_{e f f}=\Gamma v^{z}
$$

with $\Gamma \sim \phi_{0} \sqrt{\frac{R}{m}} e^{-m R}$. Thus there are two low energy states with a splitting $\sim \sqrt{R} e^{-m R} \rightarrow 0$ as $R \rightarrow \infty$.

For large but finite $R$, the energy eigenstates are eigenstates of $v^{z}$. But if the system is prepared in one eigenstate of $v^{x}$, it takes a very long time (of order $\frac{1}{\Gamma}$ ) to tunnel to the other eigenstate.

Physically, the operator $v^{z}$ adds or removes a vison from the hole of the annulus. The two eigenstates of $v^{x}$ correspond to a vison being either present or absent from the hole. In the confined phase, the "hole" spin is frozen in the up direction. The vison is therefore condensed in the hole, as it is in the rest of the sample. In the deconfined phase, a trapped vison (the "hole" spin in an eigenstate of $v^{x}$ ) stays in the hole for a time that diverges exponentially as the sample radius $R$ goes to infinity.

\section{Effect of matter fields}

In the discussion above, we considered the phases of the pure $Z_{2}$ gauge theory and the topological distinction between them. We now put back the coupling to the chargons and spinon fields. In the presence of such "matter" coupling, there continues to be a sharp distinction 
between the deconfined and confined phases. However, as is well-known [15], the behaviour of the Wilson loop is no longer sufficient to distinguish the two phases once matter coupling is included. As we will see below, there is nevertheless a topological distinction between the two phases 19.

Consider the properties of the system in a cylindrical geometry. Assume that the system is in it's deconfined phase. This implies that the vison is a gapped excitation. Consequently, a vison, once trapped in the hole of the cylinder, will stay there for a long time (of order $e^{c L_{x}}$ ) as argued previously. In the state with no vison threading the hole of the cylinder, the chargons and spinons are subject to periodic boundary conditions on encircling the cylinder. If, on the other hand, a single vison threads the cylinder, the chargons and spinons are subject to antiperiodic boundary conditions. This difference in the boundary conditions leads to a slight difference between the energies of the two states (with or without a vison threading the hole). However, this energy difference vanishes in the thermodynamic limit. Thus, the ground state is two-fold degenerate in the thermodynamic limit.

To put some meat into these observations, we restrict attention to the ground states in the two topological sectors with or without a vison in the hole, and denote these as $|\uparrow\rangle$ and $|\downarrow\rangle$, respectively. The Hamiltonian when projected to this subspace may be written,

$$
H_{\text {proj }}=\Gamma \tau^{x}+h \tau^{z}
$$

where $|\uparrow\rangle$ and $|\downarrow\rangle$ are the two eigenstates of the Pauli matrix $\tau^{z}$. Clearly, the Pauli matrix $\tau^{x}$ is the operator 20] that adds or removes a vison from the hole. The first term therefore corresponds to the tunneling of the vison, with tunneling rate $\Gamma \sim e^{-c L_{x}}$ as established in the previous subsection. The term proportional to $\tau^{z}$ comes from the difference in energy between periodic and antiperiodic boundary conditions for the chargons and spinons. The dependence of the splitting $h$ on the dimensions of the system is determined by the properties of the spectrum of the chargons and the spinons. In the insulating phases of interest, the chargon is always gapped. If the spinon is also gapped, then it is easily seen that $h \sim e^{-\tilde{c} L_{y}}$. Note that this splitting vanishes exponentially in the cylinder circumference while the vison tunneling rate vanishes exponentially in the cylinder length. In a fractionalized phase with linearly dispersing gapless spinons (as happens in the nodal liquid or the $d-R V B$ state), the splitting vanishes only as $h \sim \frac{L_{x} L_{y}}{L_{y}^{3}}$. The inverse dependence on the linear system size may be guessed by scaling considerations: Indeed the low energy theory is simply a Dirac theory for the nodal spinons. This theory is critical with a dynamic critical exponent $z=1$. Consequently, the energy $h$ vanishes inversely with the linear system size. This argument may also be verified by an explicit tedious computation [21] on a rep- resentative lattice model.

The projected Hamiltonian has two eigenvalues

$$
E_{ \pm}= \pm \sqrt{h^{2}+\Gamma^{2}}
$$

Clearly, the splitting between these two levels goes to zero in the thermodynamic limit leading to two degenerate ground states.

It is important to note that the term $h \tau^{z}$ which arises due to the presence of matter coupling explicitly breaks the topological symmetry discussed in the previous subsection. Indeed, in the restricted space above, the topological symmetry is implemented by the operator $\tau^{x}$. This no longer commutes with the Hamiltonian when matter fields are present. However, the commutator goes to zero as the system size goes to infinity. Thus, we may view the operation of threading a vison through the hole as becoming a good (topological) symmetry in the thermodynamic limit, which is then spontaneously broken. While this is, in principle, a correct point of view, it is not entirely satisfying.

The more crucial point to note is that there are two distinct topological sectors in the cylinder (with or without a vison) in the deconfined phase even in the presence of the chargons and spinons. This is simply the statement that a trapped vison stays there forever in the deconfined phase. In the confined phases, on the other hand, a trapped vison is absorbed by the vison condensate, and is very quickly lost. Therefore, there is no topological quantum number labelling the states (other than those associated with any conventional broken symmetry that may be present).

It is also useful to consider the system in an annulus geometry with a finite-sized hole at the center. Here again, in the deconfined phase, a vison that is trapped in the hole stays there forever (when the outer radius of the annulus goes to infinity). However, now there is a finite energy difference between the states with and without a trapped vison due to the change in the boundary conditions on the chargons and spinons upon encircling the hole. Thus the inability of the trapped vison to escape is really the hallmark of the fractionalized phase. The experiment proposed in Ref. 14 that we elaborate on in Section VIII probes precisely this property.

Before concluding this section, we note that a ground state degeneracy of four on a torus was suggested [22] to exist for certain states described by specific RVB wavefunctions. The same result was shown [4] to obtain in the phases of frustrated spin models that show fractionalization. In these fractionalized phases, there are neutral spin-1/2 excitations that have Bose statistics. Evidently, in this case, fractionalization has liberated the spin from the Fermi statistics of the electron. Despite the similarity in the ground state degeneracy, the topological order that characterizes this phase is distinct from that of the phases of primary interest in this paper. This may be 
seen by using gedanken flux-trapping experiments of the kind discussed in Ref. [14] (see also Ref. [11).

\section{LAYERED SYSTEMS}

Among the many unusual properties of the cuprate materials is the stark difference between the in-plane and c-axis transport. Both at optimally doped and in the slightly underdoped regime, the normal state often exhibits "metallic" in plane transport - with the resistance dropping upon cooling - which co-exists with insulating caxis transport. As emphasized by Anderson [23], this behavior is difficult to reconcile with a conventional Fermi liquid picture of the normal state, particularly in the low temperature limit (accessed by suppressing the superconductivity with strong field) where in-plane coherence of Landau quasiparticles would be expected to eventually lead to coherenct c-axis motion as well. Motivated by this puzzling behavior, we consider in this section issues of fractionalization in an anisotropic layered system. Quite strikingly, we argue that two distinct fractionalized phases are possible - one which exhibits deconfinement of spinons and chargons in all three spatial directions, and another quasi-two-dimensional fractionalized phase in which the spinons and chargons are deconfined within each layer but cannot propagate coherently between layers. In this section we restrict attention to zero temperature, turning briefly to the effects of thermal fluctuations in Section [V].

For simplicity, we will follow the strategy adopted in Section II, and initially consider the pure $Z_{2}$ gauge theory - appropriate to the layered geometry - before incorporating the spinons and chargons into the theory. To this end, consider the Hamiltonian for a $Z_{2}$ gauge theory defined on a $3 d$ cubic lattice appropriate to an anisotropic layered system:

$$
\mathcal{H}=-K_{x y} \sum_{P_{x y}} \prod_{P_{x y}} \sigma_{r r^{\prime}}^{z}-K_{\perp} \sum_{P_{\mu z}} \prod_{P_{\mu z}} \sigma_{r r^{\prime}}^{z}-h \sum_{\left\langle r r^{\prime}\right\rangle} \sigma_{r r^{\prime}}^{x}
$$

Here the first term is a sum over all plaquettes in the $x-y$ plane (normals along the z-axis) and the second term is a sum over all other plaquettes (normals lying in the $x-y$ plane, with $\mu=x, y)$. For simplicity we have taken the transverse field strength to be the same for all links of the $3 d$ spatial lattice.

As defined, this Hamiltonian depends on just two dimensionless parameters, $K_{x y}$ and $K_{\perp}$ measured in units of the transverse field $h$. The ground state phase diagram in this two-dimensional space of couplings can be readily inferred by considering various simplifying limits. For example, when $K_{\perp}=0$ the trace over $\sigma^{x}$ on the interlayer links can be trivially performed, and the model reduces to a set of decoupled $2+1$ dimensional gauge theories, one for each layer. Then, each layer has two phases - a confined phase for small $K_{x y}$ and a deconfined phase for large $K_{x y}$, as depicted schematically in Fig. 6. Away from the intervening transition, one expects the distinction between these two phases to survive for small non-zero $K_{\perp}$. In both phases, vison loops proliferate between the layers, so that the spinons and chargons which carry the $Z_{2}$ charge cannot move coherently along the $c$-axis. For small $K_{x y}$ the vison loops can also freely penetrate the layers, so that spinons and chargons are confined in all spatial directions. But the phase with large $K_{x y}$ (and small $K_{\perp}$ ) is most unsual: Since the interlayer vison loops are expelled from the layers, the in-plane motion of the spinons and chargons is coherent, but they are nevertheless confined along the $c$-axis.

To see how this unusual quasi two-dimensional deconfined phase survives with small non-zero $K_{\perp}$, we consider other limiting regimes of the phase diagram. Along the diagonal with $K_{\perp}=K_{x y} \equiv K$, the $Z_{2}$ gauge theory Hamiltonian describes an isotropic three-dimensional situation whose phase diagram is well understood - there is a first order transition at $K=K_{c}$ of order one separating the fully confined phase at small $K$ from a threedimensional deconfined phase. In the deconfined phase all large vison loops are expelled, and the spinons and chargons can propagate coherently in all three directions.

Now consider the limit of infinitely large $K_{x y}$. When $K_{x y}=\infty$, the $Z_{2}$ flux is forbidden from penetrating the $x y$ plaquettes (thereby restricting the vison loops to lie between successive layers). It is therefore possible to choose a gauge in which $\sigma^{z}=1$ on all links lying in the $x y$ plane. The system then decouples into a set of $2 d$ sub-systems, which live between adjacent layers. Consider specifically the Hamiltonian for a single such $2 d$ subsystem, which depends on the gauge fields living on the interlayer links which can be labelled conveniently by a $2 d$ square lattice of sites denoted $\boldsymbol{r}$ :

$$
\mathcal{H}_{2 d}=-K_{\perp} \sum_{\left\langle\boldsymbol{r} \boldsymbol{r}^{\prime}\right\rangle} \sigma_{\boldsymbol{r}}^{z} \sigma_{\boldsymbol{r}^{\prime}}^{z}-h \sum_{\boldsymbol{r}} \sigma_{\boldsymbol{r}}^{x}
$$

Notice that the plaquette product term has reduced to a near-neighbor Ising coupling in this sub-system Hamiltonian. Indeed, $\mathcal{H}_{2 d}$ is precisely a $2 \mathrm{~d}$ transverse field quantum Ising model, which exhibits two phases as the ratio $K_{\perp} / h$ is varied. The two phases are separated by a $2+1$-dimensional Ising phase transition. It is clear that the interlayer vison loops of the original anisotropic gauge theory, are simply domain walls separating regions with positive and negative Ising ordering, $\sigma^{z}= \pm 1$. In the ferromagnetically ordered phase of the transverse field Ising model with large $K_{\perp}$ the interfacial energy is nonvanishing. It follows that large interlayer vison loops are excluded - this is the $3 d$ deconfined phase as depicted in Fig. 6. But for small $K_{\perp}$ in the paramagnetic phase of the Ising model, the interfacial energy vanishes. In this 
case, the interlayer vison loops unbind and proliferate. This is the anisotropic quasi-2 $d$ deconfined phase (discussed above at large $K_{x y}$ and $K_{\perp} \rightarrow 0$ ). For large but finite $K_{x y}$ both deconfined phases will continue to exist. Piecing together the above results, one arrives at the final phase diagram for the anisotropic layered $Z_{2}$ gauge theory, as drawn schematically in Fig. 6.

In passing we note that phases very similar to the decoupled layered phase discussed above have been considered in other contexts in the literature. For a $U(1)$ lattice gauge theory, precisely such a phase was argued to exist when the spatial dimension of each layer is at least three in Ref. 24. In a different context, recent work 25] has examined the stability of "decoupled Luttinger liquid" phases in quasi one dimensional systems. In the context of cuprate physics, the possibility of such decoupling of the layers has been emphasized by Anderson and coworkers 23].

\section{Quasi-2d deconfined}

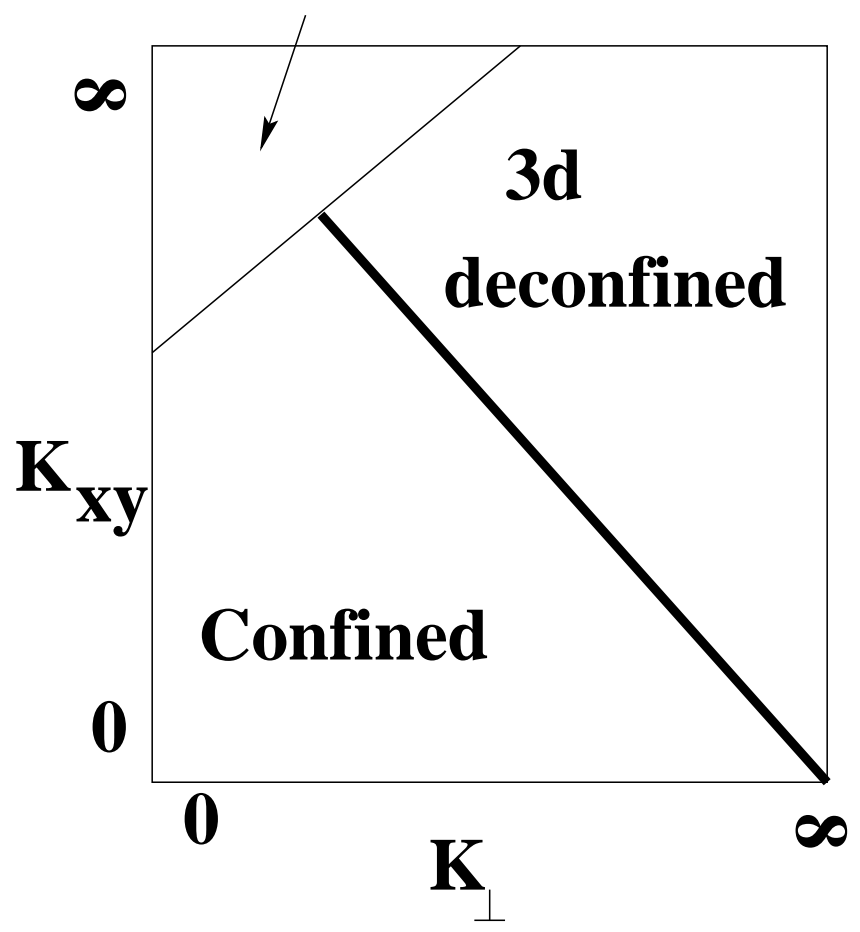

FIG. 6. Schematic phase diagram of the $Z_{2}$ gauge theory in a layered geometry. The solid line is a first order phase transition.

It is illuminating to briefly consider the topological ordering that characterizes the three phases. In the $3 d$ deconfined phase, since the vison loops are fully expelled, one expects a two-fold ground state degeneracy when periodic boundary conditions are imposed in any one of the three spatial directions, say along the $c$-axis (with open boundary conditions along the other two directions). As discussed for the $2 d$ gauge theory in Section II above, in the thermodynamic limit the two states correspond to the presence or absence of a vison loop threading the "hole" in the cylinder. When the transverse linear dimension $L$ is finite, there will be a small splitting of order $\exp \left(-c L^{2}\right)$, due to the tunnelling of an interlayer vison loop. More generally, in a $d$-dimensional deconfined phase, one expects a tunnel splitting varying as $\exp \left(-c L^{d-1}\right)$. As emphasized by Wen [5,10], the power in the exponent is particular to topological ordering, and should be contrasted to the $\exp \left(-c L^{d}\right)$ splitting between the two states of a model with a local order parameter such as the $d$ - dimensional transverse field quantum Ising model. When periodic boundary conditions are taken along all three spatial dimensions, the ground state degeneracy in the $3 d$ deconfined phase is of course $2^{3}=8$.

In the confined phase of the $3 d$ gauge theory the ground state is unique independent of the boundary conditions indicative of an absence of any topological ordering. But the topological ordering that characterizes the quasi- $2 d$ deconfined phase is somewhat subtle. With periodic boundary conditions only along the $c$-axis the ground state is unique, due to the proliferation and condensation of interlayer visons in this phase. This can also be understood more formally as follows: Consider the operation $\sigma^{z} \rightarrow-\sigma^{z}$, which changes the sign of all the interlayer bonds between (any) two adjacent layers. This is the precise equivalent for the $c$-direction of the operator $\mathcal{P}$ introduced in Eqn. 9 of Section II, and clearly changes the sign of the $Z_{2}$ flux enclosed by any curve that encircles the cylinder. Thus this operation adds or removes a vison from the hole of the cylinder. As before, it commutes with the full Hamiltonian. But notice that in the $K_{x y} \rightarrow \infty$ limit, this transformation is simply a global Ising spin flip for the $2 d$ interlayer Hamiltonian given in Eqn. 22. In the quasi-2 $d$ deconfined phase, the interlayer quantum Ising models are disordered. This implies that the ground state is invariant under the operation of threading a vison through the hole of the cylinder, and is hence unique.

Next consider the topological order in the quasi- $2 d$ deconfined phase when periodic boundary conditions are imposed in the plane, say just along the $y$ direction. To understand the resulting ground state degeneracy, it is simplest to first consider a model with two layers only, which can be conveniently visualized as two concentric cylinders with $y$-periodic boundary conditions around the cylinder. Moreover, we specialize to the $K_{x y} \rightarrow \infty$ limit which precludes visons loops from penetrating either layer. One then expects that there should be $2^{2}=4$ low energy states which belong to topologically distinct sectors. These are distinguished by the presence or absence of a vison loop threading through the bore of ei- 
ther concentric cylindrical shell. To establish that these four states are in fact degenerate (in the thermodynamic limit) first note that the symmetry operation which adds a vison to both shells simultaneously (implemented in each layer as in Eqn. 9 of Section II), commutes with the two-layer gauge theory Hamiltonian. This implies that these four states are in any case pairwise degenerate. It remains to establish, though, that the state with no visons has the same energy as the state in which (only) one of the two cylindrical shells has a threading vison. To see this, note that the operation which threads a vison through one layer only is equivalent (at $K_{x y}=\infty$ ) to changing from $y$-periodic to antiperiodic boundary conditions in the interlayer $2 d$ quantum Ising model of Eqn. 22. Since this Ising model is in it's disordered phase in the quasi-2 $d$ deconfined phase, the energy change will clearly be exponentially small in the cylinder diameter $\left(\exp \left(-c L_{y}\right)\right)$. This vanishes in the thermodynamic limit, thereby establising the degeneracy of all four states. For a layered system with $N$ layers, the ground state degeneracy with $y$-periodic boundary conditions in the quasi- $2 d$ deconfined phase is simply $2^{N}$.

Upon inclusion of the spinon and chargon matter fields which carry $Z_{2}$ charge, the nature of the topological ordering effects the inter and intra-layer confinement. In the $3 d$ deconfined phase the chargons and spinons can propagate coherently in all three spatial directions. As before, with periodic boundary conditions, they are sensitive to the presence or absence of visons trapped in the holes. In the confined phase free spinons and chargons cannot exist. But in the quasi- $2 d$ deconfined phase although the spinons and chargons can propagate coherently in-plane, they are confined to live in one layer only. The inter-layer $c$-axis transport of the chargons and spinons is fully incoherent. In this quasi- $2 d$ phase it is possible to integrate out the gauge fields living on the vertical links (trivially so when $K_{\perp} \rightarrow 0$ ). At second order in the ratio of the interlayer spinon and chargon hopping amplitudes to the transverse field, $h$, one generates inter-layer electron and pair hopping terms as well as inter-layer magnetic exchange interactions. As the chargons cannot propagate along the $c$-axis, one would expect qualitatively different inter and intra-layer charge transport at finite temperatures in this novel quasi- $2 d$ fractionalized phase. We now turn to a brief discussion of finite temperature effects.

\section{FINITE TEMPERATURE EFFECTS}

In previous sections, we have discussed a precise theoretical characterization of quantum phases (in $d \geq 2$ ) where the electron is fractionalized. There is a sharp distinction between fractionalized and un-fractionalized phases at zero temperature. Does this sharp distinction survive at finite non-zero temperatures? One normally thinks of fractionalization in terms of the spectrum of excitations of the Hamiltonian describing the system. It is then not clear what meaning it has at finite temperature. However, characterizing the fractionalized phase by it's topological order enables us to address this issue.

We again start by considering the pure $Z_{2}$ gauge theory in two spatial dimensions. As with conventional broken symmetries, thermal fluctuations play an important role in symmetry restoration when a topological symmetry is spontaneously broken. Moreover, broken topological symmetries are likewise less robust against thermal fluctations in low dimensions. Since the visons are (gapped) point-like excitations in the topologically ordered $2 d$ deconfined phase with a finite energy gap, there will be a non-vanishing density of visons created thermally at any non-zero temperature. This will immediately destroy the topological order. The situation is loosley analogous to the quantum Ising model in $1 d$, which breaks the Ising spin-flip symmetry only exactly at zero temperature. The topological symmetry restoration due to the thermally excited excited visons is perhaps easiest to understand in the $2 d$ annulus geometry. There, at zero temperature the two topologically ordered sectors correspond to the presence or absence of a vison trapped in the hole of the annulus. Clearly, at finite temperature a vison trapped in the hole of the annulus can be thermally excited into the bulk, and can then leave the sample at the outer edge of the annulus - this process interconnects the two $T=0$ states with broken topological symmetry.

In the presence of chargon and spinons matter fields the energy cost of a vison is still finite, so quite generally the $2 d$ topologically order will be destroyed at $T \neq 0$. Nevertheless, as discussed in Ref. [14] and Section VIII, by performing measurements at "short" enough timescales it should be possible to detect the presence of the $T=0$ topological order at temperatures well below the vison gap.

Remarkably, the topological order in the deconfined phase in three spatial dimensions survives thermal fluctuations intact [7]. Since the gapped vison excitations are loops in this case, they are much more difficult to thermally excite. Indeed, the energy cost of a loop grows linearly with it's length, $L$, as does the entropy associated with the loop. Thus, at low enough temperatures the free energy tension of the loop will be positive, effectively suppressing long unbound loops. Again, this reasoning remains valid in the presence of coupling to matter fields. As the temperature is raised eventually the vison loop entropy will dominate, and the system will undergo a true finite temperature phase transition at $T=T_{c} \neq 0$ that restores the topological symmetry. For $T<T_{c}$ in this $3 d$ topologically ordered phase, the free energy of two $h c / 2 e$ magnetic monopole "test" charges will grow linearly with their separation - $h c / 2 e$ magnetic monopoles are thus confined! However, an even number of magnetic monopoles - with flux an integer multiple of 
$h c / e$ - is not a source of vison flux and so costs only a finite energy. Thus, it is only a $Z_{2}$ magnetic charge which is confined in the $3 d$ topologically ordered state. This should be contrasted with the situation in a $3 \mathrm{~d}$ superconductor, which confines magnetic monopoles with any magnetic charge - a $U(1)$ magnetic confinement.

Finally, we address the effects of thermal fluctuations in the quasi- $2 d$ deconfined phase that can occur in a layered system (such as the cuprates). Here, the topological order is due to the suppression of vison loops penetrating through the layers. But the energy cost for a vison loop to pass through a layer is finite, and so will occur with non-vanishing density at at any finite temperature. Thus, strictly speaking, quasi- $2 d$ topological order in a layered system will be destroyed at any non-zero temperature, just as in the $2 d$ case.

In Ref. 14, we described in detail an experimental signature of this quasi- $2 d$ topological order, which should allow for it's detection if present in the underdoped cuprates. We elaborate on this further in Section VIII. The presence of $T=0$ quasi- $2 d$ topological order shoud also lead to dramatic differences between the low temperature in-plane and $c$-axis transport.

\section{COEXISTENCE WITH OTHER BROKEN SYMMETRIES}

If fractionalization of the electron occurs at all in the cuprates, it does so in the underdoped portion of the phase diagram. Furthermore, the fractionalized phase is presumably of the quasi- $2 d$ kind discussed at length in Section [II]. This implies that the associated topological order, strictly speaking, exists only at zero temperature. On the other hand, empirically, it is precisely in the heavily underdoped region at low temperature that a variety of conventional broken symmetry states are observed. The undoped cuprates show Neel antiferromagnetism. At intermediate doping, charge and spin stripe instabilities have been reported. Furthermore this region is often also thought to be disorder dominated. These observations raise the following conceptual questions: Can fractionalization coexist with conventional broken symmetry? Is fractionalization possible in a disordered system? Armed with the precise theoretical characterization of the fractionalized phase expounded in this paper, we now discuss the former question. The effect of disorder is considered in the following section.

Once the electron has splintered into the chargons and the spinons, various kinds of charge ordering determined by the strong Coulomb interactions between the chargons is certainly possible. Away from a doping level that is commensurate with the underlying lattice, such a charge-ordered insulating state will break lattice translational and rotational symmetries. Thus, it is obvious that fractionalization can coexist with charge order.
A more interesting issue, first raised by Balents et.al. [6], is the possibility of coexistence of fractionalization and antiferromagnetism or other kinds of magnetic order. In principle, this can be induced by interactions between the gapless spinons in the nodal liquid or d-RVB state. If such a fractionalized antiferromagnet (dubbed $A F^{*}$ ) does exist, what is it's precise distinction with the conventional Neel antiferromagnet (dubbed $A F$ )? Consider, in particular, the situation where the antiferromagnetic ordering wavevector connects two antipodal nodal points of the spinons. Then, in the presence of Neel ordering, the spinons acquire an energy gap. In this case, there would seem to be no distinction between $A F$ and $A F^{*}$ at low energies. Indeed, both phases would have gapless spin wave excitations with a linear dispersion.

The distinction is actually topological - the phase $A F^{*}$ has a topological order (and the related vison excitations) that is not shared by the phase $A F$. This may again be seen by asking for the ground state degeneracy on, say, a torus of size $L \times L$. (For simplicity, we specialize to two spatial dimensions). Due to the long range Neel order, there will be the usual tower of states [26] scaling as

$$
E_{S}=\frac{\lambda S(S+1)}{L^{2}}
$$

where $S$ is the total spin of the state, and $\lambda$ is a constant. These states should exist in both $A F$ and $A F^{*}$. But the phase $A F^{*}$ must have an additional four-fold degeneracy corresponding to trapping or not trapping a vison in each hole of the torus. Once a vison is trapped in a hole of the torus, it tunnels out at a rate $\Gamma \sim e^{-c L}$. The presence of a vison in the hole does not affect the magnons at any energy (as they are created by operators bilinear in the spinons), but it does affect the boundary conditions of the gapped spinons. This results in a difference $h \sim e^{-\tilde{c} L}$ between the energies of states with and without a vison trapped in a hole. Thus, as explained in Section [I], there are four states with a splitting that vanishes exponentially with $L$. This is to be contrasted with the tower of states above which approach zero as $1 / L^{2}$. Furthermore, all these four states will have $S=0$.

As noted above, the heavily underdoped cuprates exhibit several kinds of conventional broken symmetry - including the Neel ordering at zero doping, and charge and spin stripes at finite doping. The discussion above shows that it is theoretically possible that the fractionalization and the associated topological order coexist with these coventional broken symmetries. This is conceptually very important - the fractionalization of the electron provides a direct route to superconductivity that doesn't invoke ideas of pairing. If the heavily underdoped cuprates are fractionalized, then the Neel antiferromagnetism and the striping, while interesting phenomena, are side issues not directly related to the origin of the superconductivity. 


\section{DISORDER}

One of the remarkable aspects of superconductivity is the relative insensitivity of the Meissner effect to microscopic details, such as the symmetry of the underlying crystal structure or the presence of impurities and defects. Provided the superfluid density is non-vanishing, expulsion of magnetic flux (and of vorticity) persists. As we now discuss, the topological order that characterizes a fractionalized phase is likewise insensitive to impurity scattering. Since the essence of fractionalization is the expulsion of topological visons, just as the essence of superconductivity is the expulsion of vorticity, this insensitivity to dirt is perhaps not surprising.

We focus our discussion on the deconfined phase in two spatial dimensions. To address the issue of the stability of topological order to dirt we consider the pure $Z_{2}$ gauge theory in Eqn. 5, since coupling in the chargons and spinons will not change the essential energetics of the visons. In a spatially inhomogeneous system with impurities present, the coupling constants $K$ and $h$ in the $Z_{2}$ gauge Hamiltonian will vary randomly. The dual global Ising model in Eqn. 7 likewise becomes random - a 2-dimensional transverse field quantum Ising model with quenched random bond strengths. Upon inclusion of a doping dependent Berry's phase term in the gauge theory, the Ising bond strengths can be negative, which leads to frustration. With one electron per site the dual global Ising model is actually fully frustrated, and with randomness present will effectively be a 2 -dimensional quantum Ising spin-glass. But recall that the deconfined phase actually corresponds to the paramagnetic phase of the dual global Ising model - the phase in which the visons (the Ising spins) are gapped out rather than condensed. The Ising paramagnetic phase is clearly stable in the presence of random bonds. Frustration from the negative Ising bonds will likewise not destroy the paramagnet, and might in fact actually enhance it's stability. As mentioned above, inclusion of matter couplings will not modify this. We thereby establish the important conclusion: Topological order that characterizes electron fractionalization in two dimensions is robust and survives in the presence of impurity scattering. The $3 d$ and quasi$2 d$ deconfined phases considered in earlier sections are likewise stable to dirt. This fact is critical when one considers searching for signatures of topological order in the very underdoped cuprates, which are often riddled with defects and charge inhomogeneities (eg. stripes).

\section{TOPOLOGY-CHANGING PHASE TRANSITIONS}

In recent work we have suggested that the unusual normal state properties of the optimally doped cuprates might possibly be due to a direct quantum phase transition between a $d$-wave superconductor and a Fermi liquid. As discussed in Ref. [9], this strong coupling phase transition should be thought of as a "quantum confinement critical point". On the deconfined side of the transition the electron fractionalizes into chargons and spinons, and a subsequent condensation of the bosonic chargon leads to superconductivity. At the quantum critical point the chargons and spinons become confined together recovering the electron, and one enters a Fermi liquid phase. Unfortunately, the critical properties of this most interesting confinement transition are very difficult to access. In this section we revisit the two much simpler quantum confinement transitions mentioned in Section's II and III, and briefly address their critical properties. Since topological order present in the fractionalized phase disappears upon undergoing a confinement transition, these can be thought of as "topology changing" phase transitions.

\section{A. Two-dimensions}

Perhaps the simplest possible topology changing phase transition is the one explored briefly in Section II. For a $2 d$ cylindrical sample in a deconfined phase with a "cut" of weakened bonds running parallel to the axis of the cylinder, there are two phases: (i) A topologically ordered phase with a two-fold degenerate ground state when the bonds along the cut are strong; (ii) A phase with a unique ground state and no topological order when the bonds are weak. In the latter phase, the chargons and spinons cannot propagate coherently across the cut, and are thus deconfined on a topologically trivial manifold (the $2 \mathrm{~d}$ plane), in contrast to the former case where the chargons and spinons can be taken coherently around the cylinder.

As detailed in Section II, for the pure $Z_{2}$ gauge theory which is deep within the deconfined phase, the effective $1 d$ theory across the cut is simply the $1 d$ transverse field quantum Ising model. The quantum confinement transition corresponds to the ferromagnetic to paramagnetic transition in the Ising model, and is in the universailty class of the $D=1+1$-dimensional classical Ising model.

In the presence of gapped chargon and spinon matter fields one does not expect the universality class of this transition to be modified. But more interesting behavior becomes possible in a "nodal liquid" (or d-wave RVB) phase in which the deconfined spinons are gapless at the four nodal points. In this case one can readily write down an effective field theory that should describe the critical properties of this boundary confinement transition, by coupling the spin of the 1+1-dimensional quantum Ising model to the spinon hopping across the cut. Schematically, the effective action should take the form: 


$$
S=\int d x d y d \tau\left[\mathcal{L}_{\text {spinon }}+\mathcal{L}_{\text {Ising }}+\mathcal{L}_{\text {int }}\right],
$$

with a $2+1$ Dirac form for the spinons [27,

$$
\mathcal{L}_{\text {spinon }}=\Theta(y) \psi_{1}^{\dagger} \partial \psi_{1}+\Theta(-y) \psi_{2}^{\dagger} \partial \psi_{2},
$$

where $\psi_{1}$ and $\psi_{2}$ are nodal spinors on the two sides of the boundary, and

$$
\mathcal{L}_{\text {Ising }}=\delta(y)\left[\left(\partial_{\mu} \phi\right)^{2}+r \phi^{2}+u \phi^{4}\right],
$$

is a soft-spin $1+1$ quantum Ising model $(\mu=x, \tau)$. The (schematic) form of the boundary coupling is:

$$
\mathcal{L}_{\text {int }}=t_{b} \delta(y) \phi\left[\psi_{1}^{\dagger} \psi_{2}+\text { c.c. }\right] .
$$

When $r<0$ the Ising field picks up a non-zero expectation value, $\langle\phi\rangle \neq 0$, and the spinons can propagate coherently across the cut. For $r>0$ the Ising model is disordered, and one can integrate out the $\phi$ field, generating a spin exchange interaction across the boundary the spinons are confined on either side of the boundary, however. The boundary confinement transition occurs at $r=0$ (within mean field theory).

The critical properties can be accessed by considering a simple renormalization group transformation which rescales both spatial coordinates and time by the same factor. When $t_{b}=0$, the theory decouples into a (critical) massless $2+1$-dimensional free Dirac theory and a critical $1+1$-dimensional Ising model. The relevancy of a small interaction across the cut can then be deduced in terms of the scaling dimension of the Dirac field $\left(\Delta_{\psi}=1\right)$ and the Ising field $\left(\Delta_{\phi}=1 / 8\right)$ :

$$
\partial t_{b} / \partial \ell=\left(2-2 \Delta_{\psi}-\Delta_{\phi}\right) t_{b}
$$

Thus, the spinon hopping amplitude is actually an irrelevant perturbation, scaling to zero with eigenvalue $-1 / 8$. Being irrelevant, the transport of spinons across the cut right at the confinement transition can be deduced by working perturbatively in $t_{b}$.

\section{B. Inter-layer confinement transition}

The situation is somewhat more interesting when gapless spinons are present at the confinement transition separating the $3 d$ deconfined phase from the quasi- $2 d$ deconfined phase in an anisotropic layered situation (like the cuprates). The simplest situation to consider is that of a layered system with two layers only. To access the critical properties it is sufficient to consider the limit that $K_{x y}=\infty$, so that visons cannot penetrate through either layer. The remaining $Z_{2}$ gauge degrees of freedom live on the interlayer bonds, and are described by the $2+1$-dimensional quantum Ising model, Eqn. 22. The Ising spin is coupled to the interlayer spinon hopping. An effective field theory can be easily written down, taking a very similar form to above, except with,

$$
\mathcal{L}_{\text {spinon }}=\psi_{1}^{\dagger} \partial \psi_{1}+\psi_{2}^{\dagger} \partial \psi_{2},
$$

where now $\psi_{1}$ and $\psi_{2}$ are nodal spinors in the two layers, and

$$
\mathcal{L}_{\text {Ising }}=\left(\partial_{\mu} \phi\right)^{2}+r \phi^{2}+u \phi^{4},
$$

is a $2+1$-dimensional quantum Ising model $(\mu=x, y, \tau)$. The interaction term is (schematically)

$$
\mathcal{L}_{\text {int }}=t_{b} \phi\left[\psi_{1}^{\dagger} \psi_{2}+\text { c.c. }\right] .
$$

Once again, as above, one can consider a simple RG transformation which leaves the massless $2+1$ Dirac and critical $2+1$ Ising theories invariant. Since the boundary tunnelling interaction is now over the $2 d$ spatial plane, the eigenvalue of $t_{b}$ is modified as,

$$
\partial t_{b} / \partial \ell=\left(3-2 \Delta_{\psi}-\Delta_{\phi}\right) t_{b},
$$

with $\Delta_{\psi}=1$ as above, but now $\Delta_{\phi} \approx 0.52$ is the scaling dimension of the spin field for the $2+1$ critical Ising theory. In this case the interlayer interaction is quite strongly relevant, and one will crossover to a strongly interacting critical theory. One might be able to access this critical point by generalizing the Dirac and Ising theories to general $D=d+1$ dimensions, and expanding around a Gaussian theory perturbatively in $D=4-\epsilon$ space-time dimensions.

\section{DETECTION OF TOPOLOGICAL ORDER}

In previous sections, we have discussed how a precise theoretical characterization of fractionalized phases may be obtained through the concept of topological order. In a recent paper [14, we proposed an experiment that will directly probe this topological order. This enables a precise experimental characterization of fractionalized phases. In this section, we will discuss this experiment at length, providing more details than available in Ref. [14 and considering extensions.

The crucial property of the fractionalized phase is the inability of a trapped vison to escape from the cylinder. The effect described in Ref. [14] is a direct probe of this property and involves the following sequence of events (see Fig. 7):

(a) Start with an underdoped sample in a cylindrical geometry, with the axis of the cylinder perpendicular to the layers. In the presence of a magnetic field, cool into the superconducting phase such that exactly one $h c / 2 e$ magnetic flux quantum is trapped in the hole of the cylinder.

(b) Heat the sample to above $T_{c}$.

(c) Now turn off the magnetic field. 
(d) Cool the sample back down below $T_{c}$.

An alternate experiment is to again repeat the sequence of events (a) to (d), but now work at a fixed very low temperature and move from the superconductor into the (underdoped) insulator, and back, by adiabatically tuning some parameter.

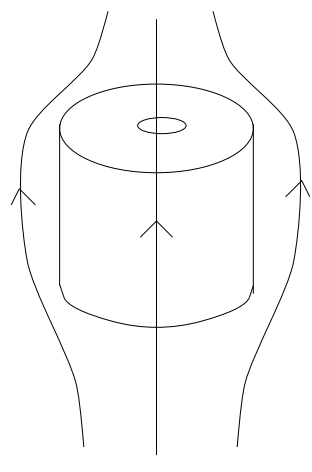

(A)

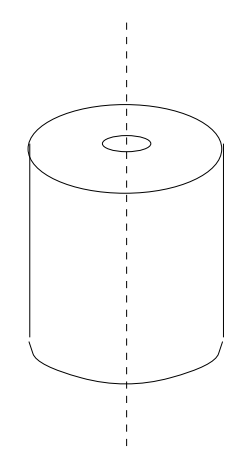

(C)

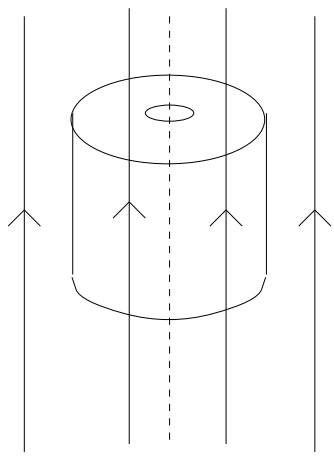

(B)

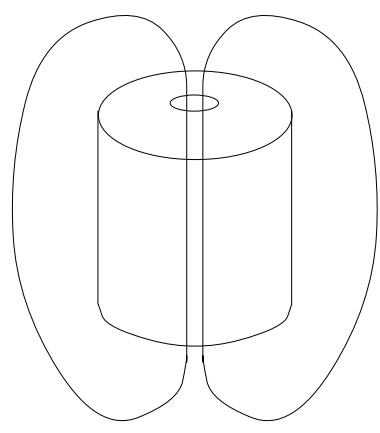

(D)
FIG. 7. The experiment to detect the topological order. The sample is superconducting in Figure A and Figure D and is "normal" in Figure B and Figure C. The electromagnetic flux is shown as a solid line. In A, a flux of $h c / 2 e$ is trapped in the hole. On moving to $\mathrm{B}$, the electromagnetic flux penetrates, but the vison (shown as dashed line) is still trapped. In $\mathrm{C}$, the sample is in zero external magnetic field, but still has a trapped vison. On moving back to the superconductor in $\mathrm{D}$, a spontaneous flux of $h c / 2 e$ appears - it's direction is arbitrary.

In the non-superconducting state at the end of step (b), the magnetic flux penetrates into the sample [28]. If, however, this state is topologically ordered, then a $Z_{2}$ flux, i.e a vison, remains trapped. (Recall that the vison is bound to the $h c / 2 e$ vortex inside the superconductor.) On turning off the magnetic field in step (c), time reversal invariance is achieved. What we have done is to prepare the sample in the non-superconducting state with a vison trapped in the hole of the cylinder. This imposes antiperiodic boundary conditions on the chargons and spinons. On moving back into the superconductor in step (d) where the chargon condenses, the vison cannot exist by itself and must nucleate an $h c / 2 e$ unit of magnetic flux. This breaks the time reversal invariance achieved in step (c). The direction of the spontaneous flux is independent of that of the initial flux.

This spontaneous appearance of a magnetic flux is a direct consequence of the inability of the trapped vison to escape in a topologically ordered phase. We have, in effect, used the superconducting state to prepare and detect the vison [29]. In particular, if the nonsuperconducting state does not have the topological order, then there will be no spontaneous flux.

In the cuprates, the fractionalization is presumably of the quasi- $2 d$ kind discussed in Section III. Thus, strictly speaking, the topological order exists only at zero temperature. In this case, if the experiment is performed by tuning some parameter to reversibly move across the superconductor-insulator phase boundary at very low temperature, a spontaneous flux is certainly expected. This is, however, much more challenging than cycling with temperature. What will be the outcome of the experiment done by varying the temperature? At a low but non-zero temperature, the trapped vison will eventually escape out of the sample in some time $t_{v}$. A spontaneous flux will be seen if the time scale for the experiment is smaller than $t_{v}$.

Decay of the trapped vison requires thermal activation across the vison gap in the bulk of the sample. Thus

$$
t_{v} \sim t_{0} e^{\frac{E_{0}}{k_{B} T}}
$$

where $T$ is the temperature, and $E_{0}$ is the vison gap. The prefactor $t_{0}$ is a microscopic time scale that depends much more weakly on temperature. Thus $t_{v}$ increases strongly with decreasing temperature.

How big is the vison gap? A lower bound on this gap may be obtained from the results of ARPES studies of the underdoped cuprates. One of the most striking features of these experiments is the absence of a quasiparticle peak in the non-superconducting state. This is indeed as expected at low temperatures below the vison gap in a fractionalized phase. The ARPES intensity continues to be broad all the way up to the pseudogap temperature $T^{*}$. This suggests that the vison gap is at least as big as $T^{*}$. In earlier work [9], we have suggested that the observed pseudogap crossover in the underdoped cuprates actually occurs at the scale of the vison gap, i.e $E_{0} \sim k_{B} T^{*}$.

A reliable estimate of the time $t_{v}$ is difficult in view of the exponential sensitivity to the ratio of the vison gap to the temperature. But the discussion above does suggest that $t_{v}$ can be enhanced enormously by enhancing 
the ratio $T^{*} / T_{c}$ and performing the experiment at temperatures close to $T_{c}$. A promising candidate material would therefore be $\mathrm{Bi}_{2} \mathrm{Sr}_{2} \mathrm{CaCu}_{2} \mathrm{O}_{8+x}$ ( $\mathrm{Bi2212}$ ). In the heavily underdoped regime when $T_{c} \sim 10 \mathrm{~K}$, a value of $T^{*} \sim 300 K$ has been reported [30].

A number of other equally robust predictions can be made for small modifications of the experiment, as also discussed in Ref. [14]. In particular, if the experiment is done with an initial flux of $\frac{n h c}{2 e}$, a spontaneous flux of $h c / 2 e$ will be observed for $n$ odd at the end of the experiment, while no spontaneous flux will be observed for $n$ even. This even/odd effect may be useful to rule out other mundane explanations of the effect, such as the presence of unknown stray magnetic fields in the sample at the end of step (c). A further observation is that the effect will not be observed if the axis of the cylinder is parallel to the layers. This is because, with quasi- $2 d$ fractionalization, vison loops are condensed in the region between the layers. A vison that is initially trapped parallel to the layers will then be quickly absorbed by this vison condensate and escape.

\section{A. Two holes and quantum tunneling of visons}

It is also extremely interesting to consider the situation where there are two holes drilled into the sample separated by a distance $l$ much smaller than the sample radius $R$. To begin with, we specialize to a strictly two dimensional system. Imagine starting in the superconducting state with a single $h c / 2 e$ flux quantum trapped in one of the two holes. Upon moving to the non-superconducting state either by heating or by other means, the magnetic flux penetrates into the sample. But again, if this nonsuperconducting state is fractionalized, the vison will be expelled from the bulk of the sample. However, in this case, the vison can tunnel back and forth between the two holes. Consider this experiment done at zero temperature by moving reversibly between the superconducting and non-superconducting phases. Then the tunneling of the vison from one hole to the other is entirely quantum mechanical. It is therefore not possible even in principle to predict with certainty which hole the vison will be in after a given amount of time. The best that can be done is to predict the probability of the vison being in any given hole. Now, on reentering the superconducting state, the vison again acquires an $h c / 2 e$ unit of electromagnetic flux. However, the resulting $h c / 2 e$ vortex can no longer tunnel so readily between the two holes. Now a measurement of the flux trapped will see a $h c / 2 e$ unit of flux in one or the other hole.

Thus the two-hole experiment offers an opportunity to probe quantum tunneling phenomena at a macroscopic scale. The superconductor is used to prepare and detect the presence of a vison. Once the non-superconducting state is prepared in a state with a vison in one hole, it evolves quantum mechanically into a state which is a linear superposition of the two states with the vison being in either hole. Moving back into the superconductor nucleates $h c / 2 e$ flux which can be used to detect the presence of a vison. The relation of the observed probability for the flux being in either hole to the original vison wavefunction (in the non-superconducting state) depends on the details of the dynamics of the system, and we will not discuss it here.

In the more complicated situation with several layers, the visons in each layer can tunnel independently between the two holes. At the end of the experiment, one frozen-in $h c / 2 e$ flux line will still be observed. This will pass through one of the two holes in each layer. The detailed shape of the flux line is an intriguing question that we leave open for the present.

\section{CONCLUSIONS}

In this paper, we have addressed a number of conceptual issues related to the possibility of electron fractionalization in spatial dimensins higher than one. Before concluding, we summarize some of the main results.

The precise theoretical characterization of a fractionalized phase is through the notion of topological order. Apart from the fractional particles into which the electron breaks apart, there are non-trivial gapped topological excitations - the visons. The full excitation spectrum therefore decomposes into different topological sectors. If a vison is initially trapped in the "hole" of a cylindrical sample that is fractionalized, it stays there forever.

Motivated by the strongly anisotropic behaviour of the cuprates in the non-superconducting states, we considered the possible fractionalized phases in a layered geometry. Interestingly, there are two kinds of fractionalized phases. In one, the system behaves like a full three dimensional solid with the chargons and spinons being able to freely propagate in all three directions. In the other phase, the different layers decouple from each other. The chargons and spinons are deconfined in each layer, but are confined in the direction perpendicular to the layers. It is this quasi- $2 d$ deconfined phase that is quite possibly relevant to the cuprates.

We also considered the effect of a non-zero temperature on the topological order. For the quasi- $2 d$ deconfined phase, the topological order does not, strictly speaking, survive at finite temperature. However, at temperature scales much smaller than the zero temperature vison gap, it is "almost" topologically ordered. In the cuprates, we have suggested [9] that the vison gap sets the scale for the pseudogap crossover.

We argued that the fractionalization could coexist with various conventional broken symmetries, and even in the 
presence of disorder. Again, the notion of topological order gives a precise characterization of ordered fractionalized phases (such as the phase $A F^{*}$ ) which distinguishes them from the corresponding ordered phases without the fractionalization.

We also briefly discussed some toy examples of quantum confinement transitions. The motivation was that precisely such a transition might possibly control the finite temperature properties of the cuprates in the region between the under and overdoped regimes.

One of the main points made in this paper is that the electron fractionalization idea provides a simple but directly testable explanation of the superconductivity in the cuprates. We now briefly review the basis for this statement.

(i) Fractionalization of the electron liberates it's charge from it's Fermi statistics. The resulting charged boson can then directly condense leading to superconductivity. This is an alternative to the pairing route to superconductivity.

(ii) Despite the alternate mechanism, the resulting superconductor is in the same phase as one obtained by condensing Cooper pairs of electrons. That this is true may appear surprising given that what is condensing is a charge $e$ boson (rather than a charge $2 e$ one). In particular, the flux quantization is in units of $h c / 2 e$. This remarkable feat is made possible by the presence of gapped topological excitations - the visons - in the fractionalized phase. Thus the existence of these excitations is crucial for the fractionalization route to superconductivity.

(iii) The experiment we propose directly detects the stability of a trapped vison in the "normal" state of the cuprates.

In view of the above, we believe that it should be possible to definitively establish or rule out the fractionalization explanation of cuprate superconductivity.

We are grateful to Leon Balents and Doug Scalapino for many stimulating discussions. We would like to thank John Kirtley for his insights as to the feasibility of the proposed vison trapping experiment. This research was generously supported by the NSF under Grants DMR97-04005, DMR95-28578 and PHY94-07194.

[1] P.W. Anderson, Science, 235, 1196 (1987).

[2] S. Kivelson, D.S. Rokhsar, and J. Sethna, Phys. Rev. B35, 8865 (1987).

[3] P.A. Lee and N. Nagaosa, Phys. Rev. B45, 966 (1992).

[4] For work on fractionalization in frustrated spin models, see N. Read and S. Sachdev, Phys. Rev. Lett. 66, 1773 (1991); S. Sachdev and N. Read, Int. J. Mod. Phys. B5, 219 (1991).

[5] X.G. Wen, Phys. Rev. B44, 2664 (1991).
[6] L. Balents, M.P.A. Fisher, and C. Nayak, Phys. Rev. B60 ,1654 (1999); L. Balents, M.P.A. Fisher, and C. Nayak, Phys. Rev. B61, 6307 (2000).

[7] T. Senthil and Matthew P.A. Fisher, to appear in Phys. Rev. B; cond-mat/9910224

[8] T. Senthil and Matthew P.A. Fisher, cond-mat/0006500.

[9] T. Senthil and Matthew P.A. Fisher, cond-mat/9912380.

[10] See for instance X.G. Wen and Q.Niu, Phys. Rev. B41, 9377 (1990).

[11] Some of these are explored in E. Demler, C. Nayak, HaeYoung Kee, Yong-Baek Kim, and T. Senthil, unpublished (in preparation).

[12] An early phenomenological discussion in the framework of the $U(1)$ gauge theory is in S. Sachdev, Phys. Rev. B45, 389 (1992); N. Nagaosa and P.A. Lee, Phys. Rev. B45, 966 (1992).

[13] This possibility was first considered in Ref. |6.

[14] T. Senthil and Matthew P.A. Fisher, cond-mat/0006481.

[15] For a review, see J. Kogut, Rev. Mod. Phys. 51, 659 (1979); F. Wegner, J. Math. Phys. 12, 2259 (1971).

[16] Some readers may find the treatment in Appendix C of Ref. 77 more illuminating.

[17] G. 't Hooft, Nucl. Phys. B153, 141 (1979).

[18] The vacuum (i.e empty space) is therefore to be regarded as a condensate of visons!

[19] This is true more generally than the specific $Z_{2}$ gauge theory considered here. In particular, for non-abelian gauge theories in $D=3+1$ with massive quark matter the ground state degeneracy in a three dimensional box with periodic boundary conditions is presumably different in the deconfined and confined phases. This precise distinction, even with matter fields, does not seem to be widely appreciated.

[20] This is simply the projection of the operator $\mathcal{P}$ to the restricted Hilbert space of the two ground states.

[21] T. Senthil and Matthew P.A. Fisher, unpublished.

[22] N. Read and B. Chakraborty, Phys. Rev. B40, 7133 (1989).

[23] For a review, see P.W. Anderson, The Theory of Superconductivity in the High-T $T_{c}$ cuprates, Princeton university Press, Princeton (1997).

[24] Y.K. Fu and H.B. Nielsen, Nucl. Phys. B236, 167 (1984).

[25] C.S. 'O Hern, T.C. Lubensky, and J. Toner, Phys. Rev. Lett. 83, 2745 (1999); V.J. Emery, E. Fradkin, S.A. Kivelson, and T. C. Lubensky, cond-mat/0001077; A. Vishwanath and D. Carpentier, cond-mat/0003036.

[26] P.W. Anderson, Physical Review, 86, 694 (1952).

[27] For simplicity, we have assumed that the interface is along the line $y=0$, i.e, along the (10) direction. For other interface directions, it may be necessary to deal with complications due to low energy "edge" modes of the $d$-wave spinons.

[28] Estimating the time scale over which the field penetrates is a simple excercise in classical electrodynamics. At long time scales the field can be shown (see, for instance, L.D. Landau amd E.M. Lifshitz, Electrodynamics of continuous media, Pergamon Press, New York (1960)) to obey a diffusion equation with "diffusion constant" $D_{B}=\frac{c^{2}}{4 \pi \mu \sigma}$. Here $\sigma$ is the conductivity, and $\mu$ is the magnetic permeability. Thus, for a sample of radius $R$, the field 
penetration occurs on a time scale $t_{B} \sim 4 \pi \mu \sigma(R / c)^{2}$. For an estimate appropriate to the cuprates, we take $\sigma \approx 10^{4}(\Omega \mathrm{cm})^{-1}, \mu \approx 1, R \approx 1 \mathrm{~mm}$ to get $t_{B} \approx 10^{-6}$ seconds.

[29] If the superconducting phase adjacent to the fraction- alized phase is itself an exotic "fractionalized superconductor" (the $S C^{*}$ phase introduced in Ref. [7]), then it cannot be used to detect a trapped vison.

[30] For a review, see for instance M. Randeria and J. -C. Campuzano, Varenna Lectures, cond-mat/9709107. 